\title{
Health-illness transition among persons using advanced medical technology at home
}

\author{
Angelika Fex, Gullvi Flensner, Anna-Christina Ek and Olle Söderhamn
}

\section{Linköping University Post Print}

N.B.: When citing this work, cite the original article.

This is the pre-reviewed version of the following article:

Angelika Fex, Gullvi Flensner, Anna-Christina Ek and Olle Söderhamn, Health-illness transition among persons using advanced medical technology at home, 2011, Scandinavian Journal of Caring Sciences, (25), 2, 253-261.

which has been published in final form at:

http://dx.doi.org/10.1111/j.1471-6712.2010.00820.x

Copyright: Blackwell Publishing Ltd

http://eu.wiley.com/WileyCDA/Brand/id-35.html

Postprint available at: Linköping University Electronic Press

http://urn.kb.se/resolve?urn=urn:nbn:se:liu:diva-63739 


\title{
Health-illness transition among persons using advanced medical technology at home
}

\author{
Angelika Fex ${ }^{1} \mathrm{MSc}, \mathrm{RN}$, Gullvi Flensner ${ }^{2} \mathrm{PhD}, \mathrm{RN}$, Anna-Christina Ek ${ }^{3} \mathrm{PhD}, \mathrm{RN}$ and \\ Olle Söderhamn ${ }^{4} \mathrm{PhD}, \mathrm{RN}$
}

\footnotetext{
${ }^{1} \mathrm{PhD}$ student Department of Nursing, Health and Culture, University West, and Department of Medicine and Health Sciences, Division of Nursing Science, Faculty of Health Sciences, Linköping University, Sweden ${ }^{2}$ Senior lecturer, Department of Nursing, Health and Culture, University West, and Department of Medicine and Health Sciences, Division of Nursing Science, Faculty of Health Sciences, Linköping University, Sweden ${ }^{3}$ Professor Emeritus, Department of Medicine and Health Sciences, Division of Nursing Science, Faculty of Health Sciences, Linköping University, Sweden

${ }^{4}$ Professor, Department of Nursing, Health and Culture, University West, Sweden, Department of Health, Faculty of Health and Sport and Centre for Caring Research Southern Norway, Faculty of Health and Sport, University of Agder, Norway
}

\author{
Corresponding author: \\ Angelika Fex \\ Department of Nursing, Health and Culture \\ University West \\ SE-461 86 Trollhättan \\ Sweden \\ E-mail: angelika.fex@hv.se
}

\begin{abstract}
This study aimed to elucidate meanings of health-illness transition experiences among adult persons using advanced medical technology at home. Since an increasing number of persons perform self-care while using different sorts of advanced medical technology at home, knowledge about health-illness transition experiences in this situation may be useful to caregivers in supporting these patients. A qualitative design was used. Five women and five men, all of whom performed self-care at home, either using long-term oxygen therapy from a ventilator or oxygen cylinder, or performing peritoneal or haemodialysis, were interviewed. Ethics committee approval was obtained. Informed consent was received from all participants and ethical issues concerning their rights in research were raised. The interviews were analysed using a phenomenological hermeneutical methodology, including both an inductive and a deductive structural analysis. This method offers possibilities to obtain an increased understanding by uncovering a deeper meaning of lived experiences through interviews transcribed as texts. The health-illness transition for adult persons in this context was found to mean a learning process of accepting, managing, adjusting and improving daily life with technology, facilitated by realizing the gain from technology at home. Further, the meaning of the health-illness transition experience was interpreted as contentment with being part of the active and conscious process towards transcending into a new state of living, in which the individual and the technology were in tune. The healthy transition experience was characterized by human growth and becoming. This study elucidates one meaning of healthillness transition experiences in relation to the use of advanced medical technology on a more generic level, independent of the specific type of technology used. A positive attitude towards technology at home facilitates the transition.
\end{abstract}

Keywords: acceptance, high-tech care, lived experience, phenomenological hermeneutics, self-care 


\section{Introduction}

Long-term changes in health and illness create a process of transition, linked to shifts in selfcare ability (1). Since patients in transition tend to be vulnerable to risks that may affect their health, facilitating transitions is in focus in the discipline of nursing (2). This demands a knowledge of general patterns of healthy transitions $(1,3,4)$.

Already in 1986, Chick and Meleis (1) stated that technological advances in medicine were subjecting persons to transitions that had not even been dreamed of a few decades earlier. Today, an increased number of chronically ill persons use advanced medical technology for self-care at home $(5,6)$, such as oxygen equipment for long-term treatment $(7)$, ventilators $(8$, 9 ), or equipment for blood or peritoneal dialysis (10). Self-care at home in this context means possibilities to enhance one's quality of life compared to treatment in hospital $(11,12)$, but is also the result of health reforms aimed at reducing the length of hospital stay $(13,14)$.

The concept of transition has been developed within nursing for more than thirty years $(4,15)$. A transition is characterized by an unstable passage between two more stable periods; a time span with an endpoint, a period of instability and confusion, and an ending with a new beginning of stability $(1,16)$. There are different types of transitions along the health-illness continuum, implying the end of an earlier life situation. Examples are the process from being healthy to critically or chronically ill, recovering from disease, or going from hospital to outpatient and home care (2).

Essential interrelated properties of transition experiences, i.e. the nature of transitions, are identified as awareness, engagement, change and difference, time span and critical points and events. Personal conditions, like those in the community and society, can facilitate or inhibit the transition process. Outcome indicators for a healthy transition, besides mastery of the new situation and fluid integrative identities, comprise indicators of feeling connected, interacting, being situated, and developing confidence and coping (4).

In order to offer support it is essential that the nurse understand the transition process (2), and only a person who experiences transition can describe it from the inside $(1,15)$. Chronic and lifestyle-related diseases create an increased need for the use of advanced medical technology at home in most industrialized countries $(5,14)$. However, no single study has been found that focuses on self-care in relation to the use of advanced technology on a more generic level, independent of the specific type of technology used, or on the meaning of transition in this context. The aim of this study was to elucidate meanings of health-illness transition experiences among adult persons using advanced medical technology at home.

\section{Method}

\section{Setting and participants}

Chronically ill patients with respiratory or kidney disorders are often required to use different sorts of advanced medical technology on a long-term basis. In order to access potential participants, contact was made with four nurses who cared for these types of patients at a hospital in western Sweden. With the aim of returning to a normal lifestyle, patients were by their physicians and nurses encouraged in taking technology home for self-care. For patients in need for dialysis, it was a choice between in-centre treatment and self-care at home. Most patients in need for long-term oxygen or a ventilator had no option but taking technology 
home, if they wanted that kind of treatment. All patients were trained and got their knowledge and skill evaluated by their nurses, first at hospital and later at the beginning in the patients' homes. Self-care was performed in regularly interaction with professional caregivers, including permanent access to nursing support.

After receiving verbal and written information about the study, the nurses were asked to give a letter containing information and an invitation to participate to patients who fulfilled the inclusion criteria: 1) performing self-care at home involving the long-term use of oxygen from a cylinder or ventilator, or performing blood or peritoneal dialysis, and 2) having the strength and ability to participate in an interview.

Ten of eleven persons who were invited to participate indicated their willingness to be interviewed. According to Dahlberg et al. (17), this sample size is appropriate in a reflective lifeworld study like this. Those who were willing to participate consented by sending their names, addresses and telephone numbers in a postage-paid reply envelope that had been provided by the nurses along with the initial information letter. These persons were then contacted by telephone, and were later interviewed in their homes by the first author.

The ten participants were between 37 and 83 years of age; five of them were under 65 years. Five were women, and seven lived with a partner at home. The study group consisted of five blue-collar workers, four white-collar workers, and one individual with no occupation. Just one was employed, and one had moved to Sweden from another northern European country. Before their technical needs at home became obvious, all participants had been treated with the prescribed technology in hospital, and were later instructed and trained by nurses in how to use it at home. Further characteristics of the participants are shown in Table 1.

\section{Interviews}

Conversational interviews were performed in the participants' homes in the summer of 2006. After some questions concerning background factors a main open-ended question was asked, focusing on the use of technology at home in daily life in connection with the prescribed treatment. This main open question was followed up with additional elucidating questions such as "How?", "What do you mean by that?", "What did you do?" etc. The interviews were tape-recorded and lasted about 45 minutes, ranging from 38 to 73 minutes.

\section{Ethical considerations}

The study was approved by the Research Ethics Committee Western Sweden, Göteborg University (Registration No. 145-06). Informed consent entailed the participants registering their agreement by providing their names, addresses and telephone numbers in a postage-paid reply- envelope they had received from the nurses. Each participant's understanding of the written patient information was followed up during the phone call while planning the interview, and before the interview in the participant's home. Ethical issues concerning the participant's rights in research (18) were raised simultaneously. 
Table 1 Characteristics of the participants

\begin{tabular}{clcl}
\hline Participant & Medical technology at home & $\begin{array}{c}\text { Years with } \\
\text { disease }\end{array}$ & $\begin{array}{l}\text { Experience with } \\
\text { technology at home }\end{array}$ \\
\hline A & ventilator, oxygen cylinder and & 25 & ventilator 10 months \\
& concentrator, tracheostomy vacuum & & \\
& pump & & \\
B & dialyser for HD & 20 & 2 years \\
C & cycler for PD & 4 & 1 year 4 months \\
D & dialyser for HD & 20 & 2 years 6 months \\
E & dialyser for HD & 5 & 4 years 6 months \\
F & oxygen cylinder and concentrator & 20 & 5 months \\
G & oxygen cylinder and concentrator, & 10 & cyl./conc. 10 years \\
& liquid oxygen & & liquid 5 months \\
H & cycler for PD & 4 & 3 years 6 months \\
I & oxygen cylinder and concentrator & 7 & 4 months \\
J & manual set for PD, & 4 & 8 months \\
& device for warming fluids & &
\end{tabular}

HD: haemodialysis; PD: peritoneal dialysis.

\section{Phenomenological hermeneutics}

A phenomenological hermeneutical method inspired by the philosophy of Ricoeur (19) and developed for healthcare research by Lindseth and Norberg (20) was used to reveal meanings of lived experiences of health-illness transition in the present context. According to Ricoeur (19), there is a dialectic movement between the understanding of the whole and the parts of a text, and between what the text is saying semantically and what it is talking about, i.e. the possibilities opened up by the hermeneutic interpretation. Interpretation means to disclose the meanings in the text. It is a movement from understanding to explanation, and further to comprehension. Lindseth and Norberg's (20) phenomenological hermeneutical method offers possibilities to obtain an increased understanding by uncovering a deeper meaning of lived experiences through interviews transcribed as texts.

The interviews were transcribed verbatim and analyzed by following the three major steps set out by Lindseth and Norberg (20): naïve reading, structural analyses and comprehensive understanding.

\section{Nä̈ve reading}

The entire text was read several times and with an open phenomenological attitude, in order to grasp the overall meaning of transition. A first conjecture, i.e. naïve understanding, was formulated of the meaning of health-illness transition experiences among persons using advanced medical technology at home, as follows: The health-illness transition for adult 
persons using advanced medical technology at home means a learning process of accepting, managing, adjusting and improving daily life with technology. This process is facilitated by realizing the gain from technology at home.

\section{Structural analyses}

A first inductive, thematic structural analysis aimed at validating the naïve understanding was performed. The whole text, interview by interview, was divided into meaning units focusing on the transition experience. Each meaning unit conveyed only one meaning of transition. The essential meaning in each meaning unit was then condensed and expressed in everyday words. Similar condensed meaning units were abstracted into sub-themes, which were assembled into themes.

To disclose various meanings, a second structural analysis was performed. Since a healthy transition means adaptedness in the new situation (4), this analysis was deductive and based on Pörn's (21-23) equilibrium theory of health and adaptedness. According to Pörn, the goals, repertoire and environment of human beings must be in equilibrium for them to experience health and adaptedness; i.e. the goal profile is realistic, the repertoire adequate and the environment appropriate, all conditions in relation to each other during the period concerned $(22,23)$. The repertoire is one's personal collection of bodily and mental abilities and knowledge of methods for actions, consisting of an inquiry system and a decision implementation stage $(21,22)$. Internal factors in the environment characterize functions of senses, energy and mobility, and external factors are of a physical, social or cultural nature (23). Examples of meaning units, condensed meaning units, sub-themes and themes for each structural analysis are shown in Table 2.

\section{Comprehensive understanding}

The last step in the analyses entailed a comprehensive understanding, in which all protocols from the naïve understanding and structural analyses were taken into account, together with the authors' preunderstanding, and interpreted as a whole.

\section{Findings}

The meanings of health-illness transition experiences among adult persons using advanced medical technology at home emerged as twenty subthemes and four themes (Table 3). The subthemes and themes are accounted for together in an integrated text, described below.

\section{Accepting advanced medical technology in daily life}

The transition process of accepting the technology and the consequences of using it at home was both emotional and cognitive. After long-term experience of hospital care, accepting the technology for self-care at home was a natural step forward for most participants. They were mentally prepared for future needs, even if this could be understood as a sign of an aggravated health situation. Acceptance came about in consultations and with support from health-care professionals, and sometimes with family and other patients who had personal experience with the technology. Consequences and alternative treatments were considered. A growing trust in personal capacity in managing the technology as well as trust in the technology facilitated the acceptance process - as did, for some participants, the opportunity to be able to 
Table 2 Examples from first inductive and second deductive structural analysis from one participant

\begin{tabular}{|c|c|c|c|}
\hline Meaning unit & Condensation & $\begin{array}{l}\text { Sub-theme/Theme in the } \\
\text { first, inductive analysis }\end{array}$ & $\begin{array}{l}\text { Sub-theme/Theme in the } \\
\text { second, deductive analysis }\end{array}$ \\
\hline
\end{tabular}

Although you are bound, certainly, you still have more freedom compared to... the need to go to the hospital three times a week.

Since I have been working with machines all my life...it is not frightening for me, like for other people who back out because they don't think they can manage...Alarms don't make me nervous, I just examine why, because there is always a reason and then you have to fix it.

And I don't have to dialyse every other day, I can skip to four days, if I want to go somewhere. Take it (dialyse) just before and just when I come back, but for a few more hours, I mean I'm able

to... adjust it to how I feel...I know from experience that that's OK.

And I had to increase the garbage collection, because of all the used infusion bags... but since I'm over 65 years old, there is no economic compensation. So we found the solution of returning it to the person who (regularly) delivers the new equipment, he gets those two boxes in return, and we are spared more frequent garbage collection and fees.
Dialysis at home means being bound, but the freedom in departing from hospital care predominates.

Being experienced in technology from work, E has knowledge of and trust in it. With alarms, he seeks systematically and calmly for errors to measure.

For special events, $\mathrm{E}$ is able to depart from routine and adjust technology to activity and health.

Dialysis at home brings extra costs for garbage. E had the idea of returning the garbage with the person who delivers new equipment, and was thus spared extra fees.
Realize the gain with technology

Accept technology

Manage technology cognitively, emotionally and practically through experience

Manage technology

Adjust technology to activity and health Adjust technology

Find logistical, economical solutions Improve technology
Consider benefits and disadvantages

Decision ability

Understand technology and repair failures calmly and

systematically

Cognitive capacity, inner environment, decision ability, action ability

Adjust technology to activity and health

Decision ability, action ability

Find logistical, economical solutions

Decision ability, action ability 
Table 3 Sub-themes and themes in the structural analyses

Sub-themes

Accept by experience

Accept by feeling trust

Accept by realizing the gain

Accept technology without reservation

Accept being bound in time and space

Manage technology cognitively, manually and emotionally

Manage to live healthy

Manage by experience, training and professional support

Develop bodily knowledge

Integrate technology into daily life activities

Manage advanced planning

Manage by having a positive attitude

Manage by receiving support from significant others

Create new meaning despites technology

\section{Adjust home to technology}

Adjust technology to health, activity and experience

Adjust activity to technology

Find practical solutions in the home

Develop technology practically and aesthetically

Struggle for better circumstances

\section{Themes}

Accept technology in daily life

Manage daily life with technology

Adjust oneself and adjust technology

Improve technology in daily life 
return to hospital treatment if they did not feel their proficiency with the technology at home was sufficient. Self-care at home involves costs for things like equipment and electricity, as well as extra work due to the technology and treatment. Some participants regarded their acceptance as not involving a choice, but rather simply as a fact to accept without reservation or pondering.

For some participants, the time to be mentally prepared for the situation with technology was short. A rapidly deteriorating course of disease caused initial reluctance to use technology at home. After participants gained insight into how the situation could be dealt with, however, they were able to see the personal benefits and feel relief that their decision to accept the technology was right.

After some experience, the difficulties and benefits of daily life with technology appeared more obvious. Feelings of being bound - both in time and space, and because of other restrictions - became noticeable. Participants continuously reconsidered their situation, especially in moments of aversion to it. Realizing the gain from technology at home, experienced as good health, a sense of freedom in time and space, an enhanced ability to understand and control technology and a decreased identity as a patient, were driving forces for acceptance. Participants did not avoid using technology in front of others, and some expressed that their self-esteem remained high.

\section{Managing daily life with advanced medical technology}

Manual skills and knowledge of the specific technology were abilities required for managing daily life with technology at home, as was knowledge of the diagnosed disorder and of doctor's orders, as well as ambitions, for healthy living. Technology itself was experienced as not difficult, but demanded sufficient cognitive ability in remembering to systematically carry out the procedures. At the beginning, participants frequently required professional support tailored to their own needs, to learn how to deal with the technology and to solve and prevent practical problems. When the support was not tailored to the participants' own needs, e.g. when teaching a group of patients with quite different life-situations at the same time, it was regarded as violating and of no value. Later, access to support was needed when problems arose. Knowing that help was always available was an appreciated source of security.

With some experience, the participants' bodily knowledge improved when they could recognize signs of good and ill health and they were able to continuously evaluate their treatment. Besides managing the technology, the participants learned not to be overwhelmed by discouraging emotions, and that any despair would pass. The participants learned to plan ahead and to be prepared for the unexpected by keeping the equipment available and operational, thus preventing mishaps.

Primarily, getting used to the situation and managing daily life with technology at home were in focus. Later on, participants felt ready to broaden their horizons. However, planning for situations like bringing heavy, bulky technology on journeys proved to be so extensive that most participants avoided travelling, besides trips within their neighbourhood. The will to mobilize a positive attitude towards technology, which can be learned with professional support, facilitated the transition process. Besides this, experiencing support from significant others was another driving force in managing the situation. This support could manifest itself as help with the technology or with demanding domestic duties due to impaired strength or 
energy, but always with an acceptance of the situation and of the need to adjust social activities to the participants' technology and capacity.

\section{Adjusting oneself and adjusting technology}

Before the technology was delivered, the home had to be prepared for the use of it. Storerooms were furnished to systematically arrange and hide all equipment, and dialysis entailed specific requirements for water, drainage and electricity. In time, the technology was adjusted in relation to the participant's physical health state, activities and experiences. Time and frequency of treatment and equipment were chosen according to bodily signs and symptoms, daily living and the knowledge of what to expect in different situations. Participants were satisfied at being able to modify the technology and maintain a social life. In contrast, activities in daily life in time and space were also adjusted to the technology, and appropriate routines for daily life with technology were found. Despite the technology, participants tried to live like before, although their activities in daily life might be reappraised. Participants living with a partner could switch roles in the family, learning to appreciate less strenuous duties like cooking, and leaving the domestic duties to their partner. With some experience, the time spent on treatment could also be considered a positive opportunity for doing other things, e.g. taking care of paperwork, instead of simply being passed. With the goal of still having an active life, new interests and projects were found that fit the demands of the technology as well as the participant's impaired physical capacity. Life was experienced as good, despite some inconvenience from the technology, with hopes for more time to enjoy good health. The opportunity to see family and friends was stated as the most vital goal.

\section{Improving advanced medical technology in daily life}

With experience of using technology at home, participants became able to act in improving the technology itself as well as its consequences. Convenient solutions were invented, like a comfortable place to sit during treatment. Technical improvements concerned efforts and suggestions for making it easier to handle and less conspicuous. Participants learnt who was in charge of obtaining increased support concerning care, financing or facilitating journeys, as well as to take courage in contacting the people in power and in fighting for better circumstances. Helping fellow patients by sharing one's own knowledge and experiences was another sign of the transition process in improving daily life with technology at home.

\section{Interpreted whole}

The meaning of the health-illness transition experience among adult persons using advanced medical technology at home was interpreted as contentment at being part of the active and conscious process towards transcending into a new state of living, in which the individual and the technology were in tune. The successful and healthy transition experience was characterized by human growth and becoming. 


\section{Reflections}

The presented interpretation is, like Lindseth \& Norberg (20) stress, one of various other possible interpretations. Further, the findings will be reflected on and discussed mainly from the health-illness transition theory perspective.

\section{Learning to accept technology: the ending of an earlier life situation}

In this study, the health-illness transition (2) focuses on the mastery of daily life with advanced medical technology at home among chronically ill adult persons. The participants' medical diagnoses were usually stated several months, or even years, before their technical needs at home, and they had already experienced the transition from health to illness, and further to chronic illness. All participants were experienced with advanced medical technology in hospital. Before using technology at home, they were trained by nurses at hospitals, as well as at home in the beginning. For persons in this context, one concrete endpoint $(1,4)$ is when the technology arrives at the home. Being chronically ill, however, the participants were mentally prepared for their coming needs. The endpoint, and a critical one (4), was rather that moment when they realized and accepted that the time had come to use technology at home.

\section{Learning to manage daily life with technology: the passage}

The level of awareness influences the level of engagement (4). Managing daily life with medical technology at home is an active action that presupposes total awareness, even though the participants did not necessarily reflect on the fact that they were in transition. Efforts for advanced planning and adjustments of the technology to activities in daily life and vice versa were signs of active engagement.

Transitions are the result of a change, and result in a change (4). Besides practical procedures involving the equipment, bringing the technology home also influenced the participants' social life and home environment, which had to be adjusted in a sometimes institutional way. The family roles could change, and daily activities like shopping and cooking took on new meaning, when regarded as possibilities to see significant others. New interests and friends were also found. When maintaining a working life was impossible, this was not because of the technology itself but rather because of impairments caused by chronic disease, and age. Growing faith in one's personal abilities in handling daily life with technology, and the understanding of this ability as more considerable than expected were other signs of change and difference in the transition process. Participants sometimes felt restricted in time and space because of the daily routine the technology demanded. By recognizing the opportunity to be at home and adjust the technology to fit in with social activities, not being restricted to the routines of hospital care and being less dependent on others, they also experienced feelings of freedom.

Personal meanings, cultural beliefs and attitudes, socioeconomic status, preparation and knowledge, like community or societal conditions, can facilitate or inhibit the transition process (4). Participants' positive attitudes to life in general, and to technology as a means of facilitating life, were vital to the transition process. They felt comfortable in seeing others during treatment, but considered the equipment as a non-aesthetic feature in the home environment. Different socioeconomic status did not seem to influence the transition process. However, being locked out of working life, with regular fees for care and medicine, as well as 
increased costs for things like equipment, electricity, garbage and transportation, were noticeable. Concerning preparation and knowledge (4), most participants experienced themselves as so well trained from their time spent in hospital that their first time using technology at home was "no big deal". Permanent access to support and regular consultations with professionals were crucial facilitators for transition, however. In the latter transition process, the ability to share knowledge with less experienced fellow patients was appreciated.

\section{Learning to adjust and improve daily life with technology: the new beginning}

A healthy transition is characterized by process indicators of feeling connected, interacting, being situated and developing confidence and coping (4). These indicators were obvious in this study as well. For all participants, seeing family and friends was the most vital goal. They deeply appreciated their support in, e.g., adjusting social events in time and space to treatment, visiting during treatment also, and providing someone to talk to in a mutual friendship. Participants felt connected by support from and even friendship with nurses, physiotherapists and physicians. This positive interaction facilitated transition and self-care. When daily life with technology at home was compared to treatment in hospital, the benefits of being at home were focused on and expressed as increased freedom and an ability to depart from the hospital routine and decreased patient identity. Instead of departing from life to hospital care, treatment now constituted actions integrated into daily life. Participants developed confidence in managing life with technology. They knew the circumstances for temporarily departing from daily routines and restrictions, felt safe with the technology and prepared to manage unexpected situations as well. Giving fellow patients their support was another sign of a cumulative knowledge and coping ability.

Mastery of new skills and behaviours needed to manage the new situation and the development of a fluid yet integrative identity are outcome indicators of a healthy transition. Levels of these outcomes indicate the quality of life for persons experiencing transition (4). The more stable situation of being at a new beginning $(1,16)$ was characterized by cognitive abilities and skills (23) in managing daily life with technology at home, adjusting technology to social activities and physical health state, setting new goals $(22,23)$, finding new interests or adapting roles in former activities, handling temporary emotions of despair (23) over the situation and experiencing life as good despite technology. Even in this state of stability (22, 4), however, activities like travelling could be too much of a challenge.

The reformulated identities are fluid rather than static, or dynamic rather than stable (4). While learning to cope with technology in daily life, participants found their personal abilities to be more considerable than they had expected. Their repertoires $(21,22)$, however, may be continuously challenged by new goals and projects. Role changes in family and former activities are mentioned as symbols of changed identities. We consider feeling comfortable using technology in front of others and expressing that one's self-esteem is still high as positive signs of a retained identity. Feeling less like a patient is a reformulated identity, and may be a reward for all efforts in this context.

Phases in a transition are more likely to merge into one another than to be discrete (1). In this study, even though the learning to accept technology at home was most focused on before and at the beginning of the new situation - i.e., the ending of the earlier life situation participants occasionally reconsidered their choice of using the technology at home, in moments of aversion to the situation as well as when experiencing the gain, such as an 
increased sense of freedom. Learning to manage life with technology was focused on in the same way not only in the passage, but also at the new beginning, when new goals were set.

The meaning of the health-illness transition experience among persons using advanced medical technology at home was interpreted as contentment at being part of the active and conscious process towards transcending into a new state of living, characterized by human growth and becoming. Participants developed confidence in managing life with technology, and found their personal abilities to be more considerable than expected. Without explicitly focusing on transition, Lindahl et al. found that dependency on a home ventilator means being in continuous movement and change (24) and having the ability to rise above oneself and cross personal boundaries in order to have a good life (25). Among socially marginalized families caring for ventilator-dependent children, family members' developing competition was a source of both pride and surprise at themselves. The caregivers cited their mastery of technology and the gain in self-esteem as significant factors in their further education (26). By viewing technology in a positive way, in realizing the gain it offers as a springboard for more time to enjoy good health, the transition process in the present study was facilitated. Transcending the possible and moving beyond one's usual limits are reflected in this positive attitude. It means the choice to move beyond one's usual limits in the actual contextual situation, in addition to the capacity to imagine other possibilities (27).

Among users of home ventilators, the competence and continuity of professional caregivers, experienced in good quality of individual-adapted teaching and access to help whenever needed, were found to be vital factors for success. The ventilator- users stated the professional caregivers' confident attitude to the positive effects of ventilator treatment as the motivating factor in facilitating the adaptation process (11). Besides the need for professional support in learning to practically manage technology, our study also elucidates the use of support in learning to emotionally and cognitively manage temporary feelings of despair at the situation, such as to gain a positive attitude towards technology at home.

\section{Methodological considerations}

The phenomenological hermeneutical method is suitable for revealing a deeper meaning in narrated lived experiences $(20,28)$, e.g. transition. All participants accepted self-care with technology at home, and showed patterns of healthy transition. Perhaps the nurses who invited suitable patients showed consideration to those who may have fulfilled inclusion criteria but were still in the unstable passage phase, by refraining from giving them an invitation. For unknown reasons, one of the persons who received an invitation did not consent to participate.

It is a challenge to interview someone about a phenomenon that is unfamiliar in every day language. It would have been unsuitable to ask the participants to, e.g., describe their transition. Instead, the interviews focused on daily life with advanced medical technology, and the participants had the opportunity to talk about their feelings and thoughts the first time they experienced the technology at home, and how they gradually learned to manage daily life. In order to switch from the natural to the phenomenological open attitude towards the phenomenon $(20,29)$, open-ended questions were raised, and a permissive interview climate in which the participants could talk freely about their lived experience in the present context was strived for. All participants gave rich descriptions. 
In this method, preunderstanding is considered an advantage, reducing the risk of misunderstandings during the interviews. As we understand in relation to our preunderstanding, it is also a prerequisite for performing the analyses (20). The authors are all versed in transition theories like the health-adaptedness theory of Pörn (21-23). The first author is familiar with the use of advanced medical technology, but from an intensive care setting. The process of interpretation is not linear, as Lindseth and Norberg (20) stress. To ensure rigour the comprehensive understanding was compared with the naïve understanding and the steps in the structural analyses, commuting back and forth between the whole and the parts, and between understanding and explanation. It may be difficult for the reader to assess trustworthiness when quotations from the raw data and themes from the deductive structural analysis are not presented explicitly. However, the common thread between naïve reading, structural analyses and interpreted whole should be visible.

\section{Conclusion}

This study elucidates one meaning of health-illness transition experiences in relation to the use of advanced medical technology at home on a more generic level, independent of the specific type of technology used. The interpretation is that one meaning of health-illness transition in this context is an active learning process of accepting, managing, adjusting and improving technology. This is a conscious process of transcending into a new state of living, in which the individual and the technology are in tune. The successful and healthy transition experience is characterized by human growth and becoming. A positive attitude towards technology at home, as a simplifier for an active life allowing more years in good health, facilitates the transition, as does ongoing access to support from professional caregivers and significant others. This study, which offers insight into the transition process experienced by those using technology, is to be regarded as one contribution to the discussion on transition. Further research with this focus is needed, however. Studies that offer insight into the experiences of significant others in this context are also required.

\section{Author acknowledgements}

We are grateful to all participating patients for sharing their experiences with us, and to the nurses who mediated contact between patients and researchers. Support from the Department of Nursing, Health and Culture, University West, and the Department of Medicine and Health Sciences, Division of Nursing Science, Faculty of Health Sciences, Linköping University, is gratefully acknowledged.

\section{Author contributions}

Angelika Fex, Anna-Christina Ek and Olle Söderhamn participated in the design of the study. The first author conducted the interviews. All four authors participated in the analysis process, and in the manuscript preparation.

\section{Funding}

The study received funding from University West, Sweden. 


\section{Ethical approval}

The study was approved by the Research Ethics Committee Western Sweden, Göteborg University (Registration No. 145-06). 


\section{References}

1 Chick N, Meleis AI. Transitions: a nursing concern. In Nursing research methodology: issues and implementation (Chinn PL ed.), 1986, Rockville, Aspen, 237-57.

2 Schumacher KL, Meleis AI. Transitions: a central concept in nursing. Image J Nurs Sch 1994; 26: 119-27.

3 Meleis AI, Trangenstein PA. Facilitating transitions: redefinition of the nursing mission. Nurs Outlook 1994; 42: 255-9.

4 Meleis AI, Sawyer LM, Im E-O, Hilfinger Messias DK, Schumacher K. Experiencing transitions: an emerging middle-range theory. ANS 2000; 23: 12-28.

5 Corbett NA. Homecare, technology, and the management of respiratory disease. Cri Care Nurs Clin North Am 1998; 10: 305-13.

6 Lehoux P. Patients' perspectives on high-tech home care: a qualitative inquiry into the userfriendliness of four technologies. BMC Health Serv Res 2004; 4: 28.

7 Swedevox. Andningssviktregistret (National quality register for treatment of respiratory impairment). 2007 (Accessed in $8^{\text {th }}$ April 2009); http://www.ucr.uu.se/swedevox

8 Laub M, Berg S, Midgren B. Home mechanical ventilation in Sweden: Inequalities within a homogenous health care system. Respir Med 2004; 98: 38-42.

9 Lloyd-Owen SJ, Donaldson GC, Ambrosino N, Escarabill J, Farre R, Fauroux B, Robert D, Schoenhofer B, Simonds AK, Wedzicha JA. Patterns of home mechanical ventilation use in Europe: results from the Eurovent survey. Eur Resp J 2005; 25: 1025-31.

10 Svenskt njurregister 2008 (Swedish renal register 2008). Dialystvärsnittsundersökningen hösten 2007 (Dialysis cross-sectional study autumn 2007). (Accessed in $8^{\text {th }}$ April 2009); http://www.medscinet.net/sur/rapporter.aspx

11 Ballangrud R, Bergseth Bogsti W, Johansson IS. Clients'experiences of living at home with a mechanical ventilator. $J$ Adv Nurs 2008; 65: 425-34.

12 Ageborg M, Allenius B-L, Cederfjäll C. Quality of life, self-care ability, and sense of coherence in hemodialysis patients: a comparative study. Hemodial Int 2005; 9: 8-14.

13 Arras JD. Bringing the hospital home: ethical and social implications of high-tech home care. 1995, John Hopkins University Press, Baltimore.

14 McNeal GJ. High-tech home care: an expanding critical frontier. Crit Care Nurse 1996; 16: $51-8$.

15 Olsson K, Ek A-C. Transition: how a concept has been used in nursing science. Theoria $J$ Nurs Theory 2002; 11: 4-12. 
16 Bridges W. Transitions: making sense of life changes. ( $2^{\text {nd }}$ updated and expanded edn.) 2004, Da Capo Press, Cambridge.

17 Dahlberg K, Dahlberg H, Nyström M. Reflective lifeworld research. (2 ${ }^{\text {nd }}$ edn.) 2008, Studentlitteratur, Lund.

18 Beauchamp TL, Childress JF. Principles of biomedical ethics. ( $6^{\text {th }}$ edn.) 2009, Oxford University Press, Oxford.

19 Ricoeur P. Interpretation theory: discourse and the surplus of meaning. (6 $6^{\text {th }}$ printing). 1976, Texas Christian University Press, Forth Worth.

20 Lindseth A, Norberg A. A phenomenological hermeneutical method for researching lived experience. Scand J Caring Sci 2004; 18: 145-53.

21 Pörn I. An equilibrium model of health. In: Health, disease, and causal explanations in medicine. (Nordenfelt L, Lindahl B.I.B. eds.), 1984, Riedel publishing company, Dordrecht, 3-9.

22 Pörn I. Health and adaptedness. Theor Med 1993; 14: 295-303.

23 Pörn I. Vad är hälsa (What is health). In: Begrepp om hälsa (Concepts of health). (Klockars K, Österman B. eds.) 1995, Liber, Stockholm, 15-28.

24 Lindahl B, Sandman P-O, Rasmussen B. On being dependent on home mechanical ventilation: depictions of patients' experiences over time. Qual Health Res 2006; 16: 881-901.

25 Lindahl B, Sandman P-O, Rasmussen B. Meanings of living at home on a ventilator. Nurs Inq 2003; 10: 19-27.

26 Cohen $\mathrm{MH}$. The technology-dependent child and the socially marginalized family: a provisional framework. Qual Health Res 1999; 9: 654-68.

27 Parse RR. The human becoming school of thought: a perspective for nursing and other health professionals. 1998, SAGE Publications, California.

28 Charalambous A, Papadopoulos R, Beadsmoore A. Ricoeur's hermeneutic phenomenology: an implication for nursing research. Scand J Caring Sci 2008; 22: 637-42.

29 Karlsson G. Psychological qualitative research from a phenomenological perspective. ( $2^{\text {nd }}$ edn.) 1995, Almqvist \& Wiksell International, Stockholm. 